\title{
Complicações da Sedação e Realização da Colonoscopia
}

\author{
Complications of Sedation and Colonoscopy Performance
}

\author{
JUVENALDA ROCHATORRES NETO ${ }^{1}$, CRISTIANE JESUS SILVINO ${ }^{2}$, ANACAROLINALISBOAPRUDENTE ${ }^{3}$, FÁBIO $^{2}$ \\ RAMOS TEIXEIRA ${ }^{4}$, FELIPEAUGUSTO DO PRADOTORRES ${ }^{5}$, JÚLIO AUGUSTO DO PRADO TORRES $^{6}$
}

\begin{abstract}
1. Prof. Dr. adjunto do Departamento de Medicina da Universidade Federal de Sergipe; ${ }^{2}$ Concludente do curso de Medicina da Universidade Federal de Sergipe; ${ }^{3 .}$ Médica Residente do $3^{\circ}$ ano de Coloproctologia do Hospital Universitário de Sergipe; ${ }^{4}$ Concludente do curso de Medicina da Universidade Federal de Sergipe; ${ }^{5}$. Acadêmico do curso de Medicina da Universidade Federal de Sergipe; ${ }^{6}$ Acadêmico do curso de Medicina da Universidade Federal de Sergipe.
\end{abstract}

\begin{abstract}
TORRES NETO JR; SILVINO CJ; PRUDENTE ACL; TEIXEIRA FR; TORRES FAP; TORRES JAP. Complicações da Sedação e Realização da Colonoscopia. Rev bras Coloproct, 2010;30(3): 318-325.

RESUMO: A realização da colonoscopia pode acarretar complicações decorrentes do preparo, sedação e dos procedimentos diagnósticos e/ou terapêuticos. Esses eventos, relativamente comuns, possuem taxa de morbidade em torno de 1\% e, em geral, são transitórios e subnotificados. Objetivo: Avaliar a incidência de complicações da sedação e realização da colonoscopia, durante e imediatamente após a mesma; relacioná-las às variáveis: gênero, idade, co-morbidades, dose das drogas para sedação/analgesia, hipertensão, hipotensão, hipóxia, taquicardia, bradicardia. Método: Estudo observacional, prospectivo e descritivo, acompanhando-se a colonoscopia de 90 pacientes em clínica privada. Utilizou-se midazolan e meperidina para a sedação. Foi aferida pressão arterial, saturação de oxigênio e freqüência cardíaca durante e imediatamente antes e após o exame. Resultados: Dos pacientes examinados, 53,3\% (n=48) eram homens. A média de idade foi 48,4 anos. Para sedação utilizaram-se 2,95mg de midazolan e 29,5mg de meperidina em média. Dos inicialmente hipertensos, $11(\mathbf{4 0 , 7} \%)$ permaneceram hipertensos ao final do exame. Observou-se saturação de oxigênio menor que $90 \%$ em $16(17,8 \%)$ pacientes; bradicardia em $19(21,1 \%)$ e taquicardia em 13 $(\mathbf{1 4 , 4 \% )}$. Náuseas, vômitos e dor abdominal foram alterações presentes em $11(12,2 \%)$ pacientes e foram devido ao preparo do cólon para a colonoscopia. Nenhuma complicação importante foi verificada.
\end{abstract}

Descritores: Colonoscopia, complicações, sedação, realização, preparo.

\section{INTRODUÇÃO}

Com o surgimento das fibras ópticas na área médica, houve grande avanço tecnológico na observação do aparelho digestivo. Atualmente a videocolonoscopia destaca-se como um dos métodos mais completos de investigação das doenças colorretais, com vantagens sobre outros métodos de investigação por proporcionar a observação da mucosa colônica e muitas vezes do íleo terminal, em tempo único e de forma direta ${ }^{(1,2,3,4)}$. Porém, o exame apresenta morbidades decorrentes do preparo, sedação, realização do exame e/ou de procedimentos terapêuticos.
Entre as complicações relacionadas às drogas utilizadas para a sedação destacam-se as reações locais, como flebites superficiais no local da injeção de diazepínicos e o prurido localizado devido ao uso de meperidina. As reações sistêmicas provocadas pelas drogas são mais significantes e potencialmente mais perigosas, a maioria delas de natureza cardiorrespiratória. As mais comuns são: hipoventilação, hipertensão, hipotensão, hipóxia, taquicardia, bradicardia. Algumas podem ser potencializadas pela dor e desconforto dos pacientes, exigindo maiores doses de sedativos. Doses adicionais podem, eventualmente, levar ao agravamento da hipóxia levando à

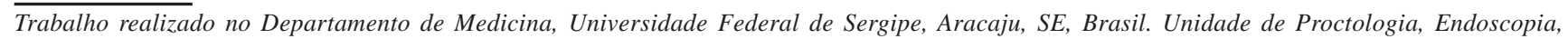
Cirurgia e Pediatria - UPEP, Aracaju, SE, Brasil. 
arritmias ${ }^{(5,6)}$, podendo mesmo determinar parada cardíaca. Esses eventos, relativamente comuns e com taxa de morbidade ao redor de $1 \%$, justificam o uso de um monitor de oxigenação sangüínea e de controle da freqüência cardíaca em todos os procedimentos colonoscópicos.

$\mathrm{O}$ índice de complicações das colonoscopias diagnósticas varia entre 0,1 a $0,5 \%$, com mortalidade de 0,02 a $0,15 \%$. Já a colonoscopia terapêutica apresenta mortalidade de 1 a $6 \%$ e morbidade de 1 a $2 \%$, atingindo até $35 \%{ }^{(7,8)}$. A hemorragia pós-polipectomia transcolonoscópica tem índice de complicação de 1 a $4 \%$, e a perfuração pode ocorrer entre 0,34 e $2,14 \%$ (7, 9,10,11). Essas complicações são relatadas na maioria dos trabalhos científicos, porém complicações menores, por serem transitórias e geralmente limitadas ao período de realização do exame e de recuperação pósanestésica, são subnotificadas.

Objetivamos, portanto, avaliar a incidência das complicações decorrentes da sedação e da realização da colonoscopia, durante e imediatamente após a mesma.

\section{PACIENTES E MÉTODOS}

Foi realizado trabalho prospectivo, observacional e descritivo, acompanhando-se 90 pacientes da Unidade de Proctologia, Endoscopia, Cirurgia e Pediatria (UPEP) durante o exame colonoscópico. O período da observação foi entre abril e agosto de 2007. As variáveis foram: gênero, idade, tempo de realização do exame, volume das drogas utilizadas para sedação/analgesia, co-morbidades, hipertensão, hipotensão, hipóxia, taquicardia, bradicardia.

Todos os pacientes receberam a mesma instrução quanto ao preparo do cólon. Na véspera do exame a dieta recomendada foi pastosa, sem resíduos e sem leite. Às 19 hs todos tomaram lactulose (4 colheres das de sopa) ou bisacodil (2 drágeas). Seis horas e trinta minutos antes do exame foi feito uso de 35 gotas, via oral, de cloridrato de metoclopramida ( $9 \mathrm{mg}$ ). E, seis horas antes foi iniciada a ingestão da solução de Manitol a 10\% (2 frascos de manitol a 20\% diluído em $500 \mathrm{ml}$ de água de coco), tomandos fracionadamente (150ml de cinco em cinco minutos). Recomendou-se maior deambulação e dieta zero após a ingestão do manitol, sendo permitido apenas ingestão de água até quatros horas antes do exame. A qualidade de cada preparo foi avaliada a partir dos dados referidos pelo examinador em um relatório, sendo considerados como referência os parâmetros estabelecidos por Vanner et al. (1990) ${ }^{(12)}$ e graduados em excelente (ausência ou pequeno volume de líquido claro no interior do cólon), bom (grande volume de líquido claro, sem resíduos), regular (resíduos fecais com possibilidade de aspiração) e ruim (resíduos fecais semi-sólidos, impossíveis de aspiração adequada).

O trabalho foi aprovado pela comissão de ética do Hospital Universitário da Universidade Federal de Sergipe.

Antes da realização do exame, foi explicada ao paciente a natureza da pesquisa, sendo solicitada a assinatura do termo de consentimento livre e esclarecido, preenchendo-se o protocolo da pesquisa junto ao paciente.

A sedação foi feita com midazolam e meperidina. Embora a dose para adultos recomendada na literatura seja de 5 a $15 \mathrm{mg}$ do midazolam e de 25 a $100 \mathrm{mg}$ da meperidina por via endovenosa ${ }^{(13,14)}$, na amostra estudada fez-se uso de $5 \mathrm{mg}$ de Midazolam $(5 \mathrm{ml})+50 \mathrm{mg}$ de Meperidina $(1 \mathrm{ml})$ diluídos em $14 \mathrm{ml}$ de água destilada. Três minutos antes do exame, foi administrada metade da dose em "bolus"; reduzindo-se a um quarto a dose em pacientes acima dos 70 anos e/ou que possuiam co-morbidades. Estes últimos foram acompanhados por anestesiologista durante o período de realização do exame. Doses adicionais foram administradas, conforme a necessidade de cada paciente, objetivando não ultrapassar $5 \mathrm{mg}$ de midazolan e 50mg de meperidina. Ao final do exame foi medida a dose total em mg de cada droga recebida por cada paciente.

Foram aferidos os valores da pressão arterial (PA), da saturação de oxigênio e da frequiência cardíaca (FC) a cada dois minutos durante o procedimento e, dois minutos antes do início e após o término do mesmo. Foram consideradas as intercorrências até uma hora após o exame e no momento da sua detecção foram novamente aferidos os mesmos parâmetros.

Os dados foram colocados em planilhas no Excel@ 2003 para análise descritiva das variáveis estudadas bem como para a confecção de gráficos e tabelas.

Os pacientes foram classificados, segundo os valores encontrados da PA em hipertensos (PA sistólica maior que $140 \mathrm{mmHg}$ e diastólica maior que 90), normotensos (PA sistólica entre 90 e $140 \mathrm{mmHg}$ e diastólica entre 60 e $90 \mathrm{mmHg}$ ) e, hipotensos (PA sistólica menor que $90 \mathrm{mmHg}$ e diastólica menor que $60 \mathrm{mmHg})^{(15)}$. 
Segundo valores da saturação de oxigênio, os pacientes foram classificados em hipoxêmicos leves (saturação entre 80 e 89\%), hipoxêmicos moderados (saturação entre 70 e 79\%) e hipoxêmicos graves (saturação menor que 70\%). Conforme valores da FC, foram classificados em taquicárdicos (FC > 100bpm), FC normal (FC entre 60 e 100bpm) e bradicárdicos (FC $<60 \mathrm{bpm})$.

\section{RESULTADOS}

Foram examinados 90 pacientes, sendo 48 $(53,3 \%)$ homens e $42(46,7 \%)$ mulheres. A idade variou de 24 a 76 anos, com média de 48,4 anos. A maioria dos pacientes $(n=54 ; 60,0 \%)$ encontravam-se entre 41 e 60 anos de idade.

A duração média do exame foi de 10,8 minutos, variando de 4 a 22 minutos. Utilizaram-se $2,9 \mathrm{mg}$ de midazolan e $29 \mathrm{mg}$ de meperidina, em média, sendo as doses máximas administradas de $5 \mathrm{mg}$ e $50 \mathrm{mg}$, e mínimas, de $1,3 \mathrm{mg}$ e $13 \mathrm{mg}$, respectivamente (Tabela 1).

Dentre as co-morbidades, $8(8,9 \%)$ pacientes apresentavam hipertensão arterial sistêmica (HAS), 4 $(4,4 \%)$ HAS associada à Diabetes Mellitus (DM), 1 $(1,1 \%)$ DM, $1(1,1 \%)$ Doença Pulmonar Obstrutiva
Crônica (DPOC) e $1(1,1 \%)$ Infarto Agudo do Miocárdio (IAM) (Gráfico 1). A maior parte $(\mathrm{n}=75$; $83,3 \%$ ) dos pacientes não apresentava co-morbidades. Como indicação para o exame observou-se que 31 pacientes $(34,4 \%)$ apresentavam dor abdominal, 22 $(24,4 \%)$ constipação, $18(20,0 \%)$ sangramento pelo ânus, $13(14,4 \%)$ dor anorretal, $9(10,0 \%)$ diarréia, 7 (7,8\%) seguimento de doença diverticular, 7 (7,8\%) doença inflamatória intestinal, $6(6,7 \%)$ seguimento de pólipo, $5(5,6 \%)$ massa abdominal, 4 (4,4\%) sangue oculto nas fezes, $2(2,2 \%)$ alternância do hábito intestinal, $2(2,2 \%)$ tumoração anal e um $(1,1 \%)$ incontinência fecal (Tabela 2).

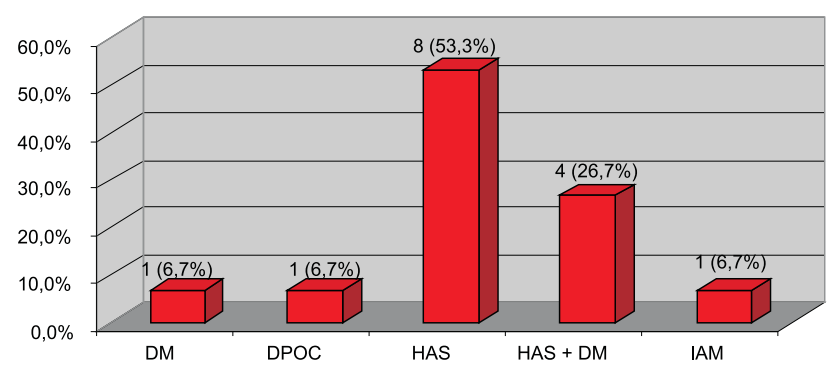

Gráfico 1 - Co-morbidades encontradas nos pacientes submetidos à colonoscopia.

Tabela 1 - Dose das drogas utilizadas na sedação/analgesia.

\begin{tabular}{lccc}
\hline Droga / Dose & Máxima & Média & Mínima \\
\hline Midazolan & $5 \mathrm{mg}$ & $2,9 \mathrm{mg}$ & $1,3 \mathrm{mg}$ \\
Meperidina & $50 \mathrm{mg}$ & $29 \mathrm{mg}$ & $13 \mathrm{mg}$ \\
\hline
\end{tabular}

Tabela 2 - Indicações das 90 colonoscopias realizadas.

\begin{tabular}{lrc}
\hline Dor abdominal & 31 & $34,4 \%$ \\
Constipação & 22 & $24,4 \%$ \\
Sangramento pelo ânus & 18 & $20,0 \%$ \\
Dor anorretal & 13 & $14,4 \%$ \\
Diarréia & 9 & $10,0 \%$ \\
Seguimento doença diverticular & 7 & $7,8 \%$ \\
Doença inflamatória & 7 & $7,8 \%$ \\
Seguimento de pólipo & 6 & $6,7 \%$ \\
Massa abdominal & 5 & $5,6 \%$ \\
Sangue oculto nas fezes & 4 & $4,4 \%$ \\
Alternância de hábito intestinal & 2 & $2,2 \%$ \\
Tumoraçao anal & 2 & $2,2 \%$ \\
Incontinência fecal & 1 & $1,1 \%$ \\
\hline
\end{tabular}


Quanto ao preparo do cólon, pudemos classificar em: excelente $(n=75 ; 83,3 \%)$, bom $(n=9 ; 10,0 \%)$, regular $(\mathrm{n}=4 ; 4,4 \%)$ e, ruim $(\mathrm{n}=2 ; 2,2 \%)$. Os pacientes com preparo ruim foram aqueles portadores de neoplasia de sigmóide $(1 ; 1,1 \%)$ e reto $(1 ; 1,1 \%)$, sendo o exame interrompido tão logo feito o diagnóstico. Um caso de neoplasia de sigmóide foi classificado como regular.

O exame teve como limite superior o ceco em $86(95,6 \%)$ pacientes, o cólon descendente em 2 (2,2\%), o sigmóide em $1(1,1 \%)$ e o reto em $1(1,1 \%)$. Nos elementos em que se atingiu o ceco, o íleo terminal foi examinado em $81(94,2 \%)$ casos.

Três $(3,3 \%)$ pacientes apresentaram vômitos após o exame e 8 (8,9\%), dor abdominal em cólica. Em ambas as situações, após administração de medicações, houve melhora dos sintomas.

Em $34(37,8 \%)$ pacientes o exame foi normal. O diagnóstico mais freqüente foi doença diverticular, com $19(21,1 \%)$ casos, seguida de pólipo em $12(13,3 \%)$, colite em $6(6,7 \%)$, angiectasia em 4 (4,4\%), ileíte em $4(4,4 \%)$, neoplasia em 3 (3,3\%). Além desses, detectou-se hiperplasia linfóide de íleo terminal (1 caso), suspeita de megacólon ( 1 caso), oxiuríase ( 1 caso), proctite (1 caso), úlceras colônicas (1 caso); além de que houve interrupção do exame interrompido em 1 caso, com distribuição percentual de $1,1 \%$ em cada uma dessas situações.

Vinte e sete $(30,0 \%)$ pacientes encontravamse hipertensos, $62(68,9 \%)$ normotensos e $1(1,1 \%)$ hipotenso no início do exame.

Dos hipertensos, 11 (40,7\%) mantiveram-se na mesma condição até o final do exame, $15(55,6 \%)$ ficaram normotensos e 1 (3,7\%) ficou hipotenso. Dos que se mantiveram hipertensos, $3(27,3 \%)$ tiveram aumento da pressão e $8(72,7 \%)$ diminuição da mesma. Nos que elevaram a pressão, houve um aumento médio de $23 \mathrm{mmHg}$ na PAS e de $11 \mathrm{mmHg}$ na PAD. Nos que reduziram a pressão, houve uma diminuição média da PAS de $12 \mathrm{mmHg}$ e da PAD de $8 \mathrm{mmHg}$.

Dos que se encontravam normotensos antes do exame, $52(83,9 \%)$ mantiveram-se na mesma condição até o final do exame, 8 (12,9\%) ficaram hipertensos e $2(3,2 \%)$ hipotensos. Dos que se mantiveram normotensos, $20(38,5 \%)$ tiveram aumento da pressão e $32(61,5 \%)$ diminuição da mesma. Nos que elevaram a pressão, houve um aumento médio de $11 \mathrm{mmHg}$ na PAS e $6 \mathrm{mmHg}$, na PAD. Nos que redu- ziram a pressão, houve diminuição na PAS de $12 \mathrm{mmHg}$ e na PAD de $6 \mathrm{mmHg}$.

O paciente que se encontrava hipotenso antes do exame tornou-se normotenso durante o mesmo.

Na Figura 1 estão relacionadas as classificações quanto à PA no início e no final do exame colonoscópico, e sua respectiva variação média nos valores da PAS e PAD.

Dos pacientes portadores de HAS, 7 (58,3\%) encontravam-se hipertensos no começo do exame, e 5 $(41,7 \%)$ normotensos. Ao final do exame, $9(75,0 \%)$ estavam normotensos e $3(25,0 \%)$ permaneceram hipertensos. Todos os pacientes portadores de hipertensão não fizeram uso do antihipertensivo no dia do exame.

De todos os pacientes examinados, $88(97,8 \%)$ se encontravam com saturação de oxigênio entre 90 e $99 \%$ e $2(2,2 \%)$ com saturação entre 80 e $89 \%$ no início do exame.

Daqueles com saturação entre 90 e $99 \%, 72$ $(81,8 \%)$ mantiveram-se na mesma condição até o final do exame, $14(15,9 \%)$ tiveram ao menos uma medida da saturação entre 80 e $89 \%$ e $2(2,3 \%)$, abaixo de $80 \%$ (Gráfico 2).

Os dois elementos que iniciaram com saturação entre 80 e $89 \%$, terminaram o exame com saturação de $98 \%$, sendo que a mínina saturação observada entre eles foi de $84 \%$.

Dos $16(18,2 \%)$ pacientes que tiveram ao menos uma medida da saturação menor que $90 \%$ durante o procedimento, $10(62,5 \%)$ encontravam-se entre 41 e 60 anos. Não houve diferença na dose das drogas utilizadas. Seis $(37,5 \%)$ pacientes possuíam alguma co-morbidade: $4(66,6 \%)$ HAS, 1 (16,7\%) DM e 1

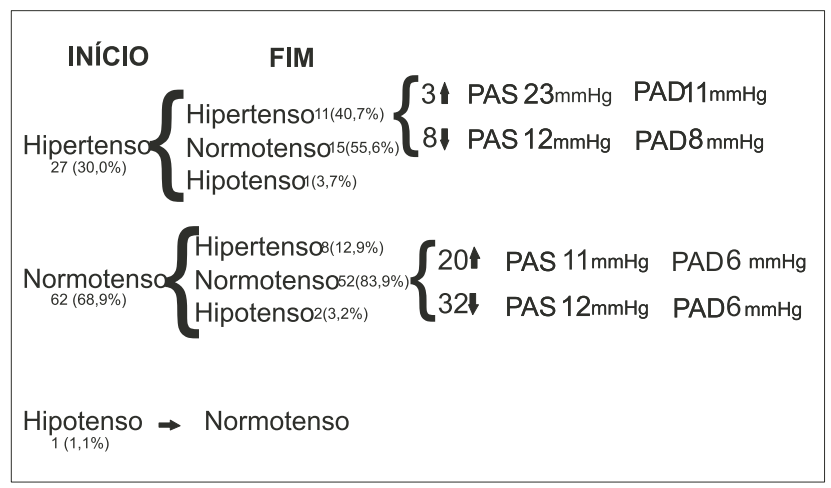

Figura 1 - Comportamento da pressão arterial e, a variação média da PAS e da PAD dos que permaneceram hipertensos $e$ normotensos até o final do exame. 
$(16,7 \%)$ DPOC. Em apenas um caso houve necessidade de usar oxigênio com máscara de Venturi a 50\%. Este já iniciou o exame com saturação de $89 \%$.

Em relação à $\mathrm{FC}$ durante a colonoscopia, 1 $(1,1 \%)$ encontrava-se com FC acima de $100 \mathrm{bpm}$ no início do exame, $87(96,7 \%)$ entre 60 e 100bpm e 2 $(2,2 \%)$ abaixo de 60.

O paciente que apresentou taquicardia regularizou a $\mathrm{FC}$ até o final do exame.

Dos elementos que estavam inicialmente com FC entre 60 e 100bpm, 55 (63,2\%) mantiveram-se na mesma condição até o final do exame, $10(11,5 \%)$ tiveram freqüência entre 101 e $120 \mathrm{bpm}, 2(2,3 \%)$ entre 121 e $140 \mathrm{bpm}$ e $1(1,2 \%)$ 144bpm. Já $17(19,5 \%)$ tiveram valores entre 40 e $59 \mathrm{bpm}$ e $2(2,3 \%)$ FC menor que 40bpm (Gráfico 3)

Os pacientes bradicárdicos no começo da colonoscopia ficaram com freqüência cardíaca normal durante o exame, pórem ao final do mesmo 1 (50\%) estava com FC de $52 \mathrm{bpm}$.

No término do exame $3(3,3 \%)$ pacientes encontravam-se taquicárdicos e $6(6,7 \%)$ bradicárdicos. Oitenta e um $(90,0 \%)$ estavam com FC entre 60 e $100 \mathrm{bpm}$.

A Figura 5 mostra a variação da FC nos pacientes que apresentaram bradicardia e taquicardia durante a colonoscopia.

\section{DISCUSSÃO}

A colonoscopia é exame de suma importância para a coloproctologia e especialidades correlatas. Várias são as indicações para realizar a colonoscopia. As contra-indicações são cada vez mais restritas e em sua maioria apenas relativas.

As indicações mais freqüentes ocorreram na seguinte sequiência: $1^{\circ}$ lugar dor abdominal $(n=24$; 27,0\%); $2^{\circ}$ lugar: constipação $(n=19 ; 21,3 \%) ; 3^{\circ}$ lugar: sangramento pelo ânus $(\mathrm{n}=14 ; 16 \%) ; 4^{\circ}$ lugar: diarréia $(\mathrm{n}=8 ; 9,0 \%)$. SILVA et al ${ }^{(16)}$ relatam incidências diferentes: em primeiro lugar as diarréias; em segundo os sangramentos anais; em terceiro, as dores abdominais e em quarto, a constipação

Apesar de ser um exame rotineiro, tem uma morbidade não desprezível, quer seja pelo preparo, sedação, realização do exame ou pela terapêutica. Muitas vezes a realização do exame é necessária em idosos e/ou portadores de co-morbidades, o que contribui para aumentar a referida morbidade.

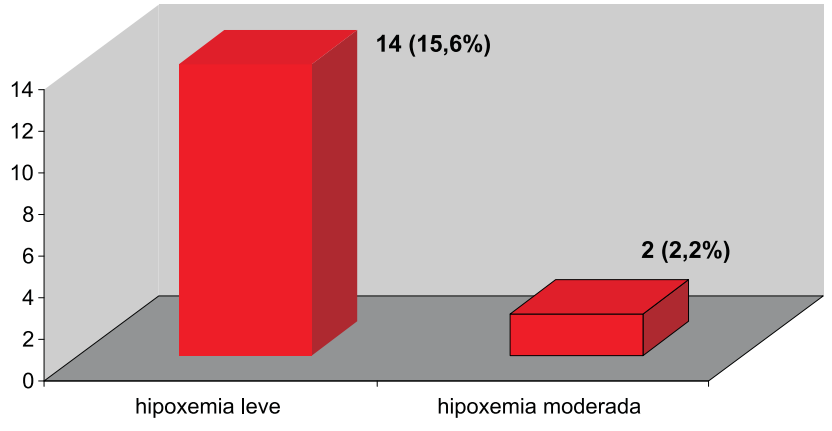

Gráfico 2 - Hipoxemia durante a colonoscopia.

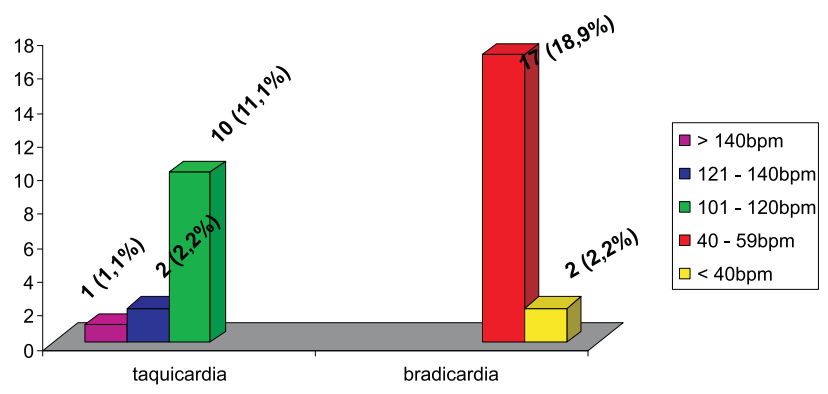

Gráfico 3 - Variação da freqüência cardíaca nos pacientes que apresentaram bradicardia e taquicardia durante a colonoscopia.

A maioria dos trabalhos científicos relacionados a complicações inerentes à colonoscopia, visa estudar complicações "maiores" como perfuração e sangramento $(7,9,10,11)$. O presente trabalho estudou principalmente as complicações "menores", em geral transitórias e subnotificadas.

As complicações relacionadas ao preparo são principalmente náuseas, vômitos, distúrbios eletrolíticos ou dor abdominal, quer pela ingestão de soluções hipertônicas com diarréia, quer pelo jejum mais prolongado ${ }^{(17)}$. Na investigação atual, $11(12,2 \%)$ pacientes apresentaram náuseas; vômitos ou dor abdominal. Apesar de incidir num número considerável de pacientes esses transtornos foram de curta duração, cedendo com medicação sintomática. Mesmo tendo havido morbidade relacionada ao preparo este foi classificado excelente e bom na maioria $(\mathrm{n}=84 ; 93,3 \%)$ dos pacientes, como relatado nos trabalhos de HABR-GAMA et al ${ }^{(18)}$.

Geralmente realiza-se a colonoscopia sob sedação com benzodiazepínicos e analgésicos opióides. São utilizados concomitantemente para potencializar a sedação. O midazolan possui efeito limitado sobre os sistemas cardiovascular e respiratório, produzindo pouca modificação na PA e apenas uma depressão transitó- 
ria do volume-minuto respiratório. Sua ação mais proeminente e facilmente quantificável é a depressão do sistema nervoso central (SNC). A meperidina causa depressão respiratória por diminuição da responsividade do SNC ao aumento da pCO2, sendo dose dependente. Às vezes causa leve bradicardia, já em altas doses pode levar a hipotensão arterial devido à liberação de histamina e depressão central dos mecanismos de estabilização vasomotora ${ }^{(19,20)}$.

Além dos efeitos colaterais referentes ao preparo e sedação, a realização do exame tem também o potencial de acarretar complicações em função da insuflação de ar no tubo digestivo, tração sobre o mesocólon e dor no paciente, desencadeando ou agravando complicações como aumento ou diminuição da $\mathrm{PA}$, bradicardia, assistolia ${ }^{(21,22,23)}$ ou levando à necessidade de maiores doses de hipnóticos e hipoanalgésicos.

Foram utilizados em média 2,9mg de midazolan e $29 \mathrm{mg}$ de meperidina, sendo a dose máxima administrada se cada droga, entre $5 \mathrm{mg}$ e $50 \mathrm{mg}$, e mínima, entre $1,3 \mathrm{mg}$ e $13 \mathrm{mg}$, respectivamente.

Houve, em função da sedação e mesmo da coexistência de hipertensão arterial, variações nos níveis de PA. Alguns pacientes com HAS e que chegaram ao exame hipertensos (todos haviam deixado de tomar o anti-hipertensivo de hábito), se beneficiaram com a sedação, tornando-se normotensos durante o exame. Porém, outros apresentaram aumento da PA, em detrimento da sedação utilizada e outros ainda diminuíram os níveis de pressão, pórem nenhum deles ficou hipotenso.

A maioria dos pacientes normotensos continuou normotensa apesar de $20(38,5 \%)$ apresentarem um aumento relativo da pressão arterial durante o exame e $32(61,5 \%)$ terem experimentado uma diminuição na referida pressão. Em função da sedação/exame três pacientes apresentaram hipotensão. A monitorização da PA durante todo o exame é fundamental para prevenir possíveis agravamentos desta morbidade. Essas alterações ocorreram numa minoria dos pacientes da amostra e foram facilmente contornáveis.

Para a realização do exame já está bem determinada a importância da utilização da oximetria de pulso com o objetivo de diagnosticar e corrigir prontamente a diminuição de saturação de oxigênio, taqui ou bradicardias, principalmente em idosos e/ou portadores de co-morbidades. A depressão do nível de consciência acarreta redução de saturação de oxigênio.
Dos 90 pacientes submetidos ao exame $16(17,8 \%)$ apresentaram queda na saturação de oxigênio abaixo dos $90 \%$, servindo como primeiro alerta para correção, seja com a utilização de oxigênio ou simplesmente solicitando ao paciente que inspirasse mais profundamente. Destes, apenas $2(12,5 \%)$ apresentaram saturação abaixo de $80 \%$. Tais intercorrências foram prontamente corrigidas, ratificando a importância da monitoração.

Antes de iniciar a colonoscopia, apenas um paciente estava taquicárdico. No início do exame mais $13(14,4 \%)$ apresentaram taquicardia, mas a sedação convencional foi suficiente para a correção. A bradicardia estava presente em $2(2,2 \%)$ pacientes antes de iniciar a colonoscopia e com as manobras de realização do exame, $19(21,1 \%)$ apresentaram queda na FC e um $(1,1 \%)$ apresentou uma freqüência de $36 \mathrm{bpm}$, revertida apenas com a desinsuflação do cólon e retirada parcial do aparelho. Ao final do exame, $6(6,7 \%)$ estavam com FC entre 52 e $59 \mathrm{bpm}, 3(3,3 \%)$ entre 102 e $106 \mathrm{bpm}$ e 81 $(90,0 \%)$ com FC normal.

Para grande parte dos exames o resultado foi normal $(\mathrm{n}=34 ; 37,8 \%)$, como relatado no trabalho de NAHAS et al ${ }^{(24)}$. O diagnóstico mais freqüente foi o de doença diverticular dos cólons $(\mathrm{n}=19 ; 21,1 \%)$, comum na faixa etária examinada, segundo trabalho de SILVA et al ${ }^{(17)}$.

Alguns pacientes apresentaram também sudorese fria associada à hipotensão que pode ter sido decorrente de hipercapnia, a capnometria não foi avaliada. Como já padronizado no serviço, pacientes com necessidade de maiores cuidados foram acompanhados desde o inicio do preparo e sistematicamente pelo anestesiologista com esquema de sedação e analgesia mais particularizado.

Apesar de as complicações "menores" parecerem pouco importantes, elas ocorrem numa frequiência razoável e se não houver monitorização adequada a morbidade poderá ser bem maior, podendo implicar em mortalidade do paciente.

\section{CONCLUSÕES}

A morbidade decorrente do preparo colônico consistiu em náuseas, vômitos e/ou dor abdominal transitórios e de pequena monta.

Apesar de ter havido variação dos níveis tensionais, da FC e da saturação de oxigênio em de- 
corrência da sedação e realização do exame colonoscópico, nenhuma complicação importante foi verificada nos pacientes estudados. Todas as intercorrências foram prontamente corrigidas: três casos de hipotensão (3,3\%), 19 de hipertensão $(21,1 \%)$,
19 de bradicardia (21,1\%), 13 de taquicardia $(14,4 \%)$ e 16 de hipoxemia $(17,8 \%)$ no decorrer do exame.

Ratifica-se a importância da monitorização com oximetria de pulso e medida da PA não invasiva durante todo o exame de colonoscopia.

ABSTRACT: The accomplishment of the colonoscopy can cause decurrent complications of the preparation, sedation and of the disgnostic and/or therapeutical procedures. These events, relatively common, around possess tax of morbidity of $1 \% \mathrm{e}$, in general, transitory and are subnotificados. Objective: To evaluate the incidence of complications of the sedation and accomplishment of the colonoscopy, during and immediately after the same one; to relate them it the 0 variable: sort, age, comorbid, amount of the drugs for sedation/analgesic, hypertension, hypotension, hypoxia, tachycardia, bradycardia. Method: Observational, prospective and descriptive study, being accompanied by it colonoscopy of 90 patients in private clinic. One used midazolan and meperidina for the sedation. It was surveyed arterial pressure, saturation of oxygen and cardiac frequency during and immediately before and after the examination. Results: Of the examined patients, $53.3 \%(n=48)$ were men. The age average was 48,4 years. For sedation they had on average used $2,95 \mathrm{mg}$ of midazolan and $29,5 \mathrm{mg}$ of meperidina. Of the initially hypertensive ones, $11(40.7 \%)$ had remained hypertensive to the end of the examination. Saturation of lesser oxygen was observed that $90 \%$ in $16(17.8 \%)$ patient ones; bradycardia in $19(21.1 \%)$ and tachycardia in $13(14.4 \%)$. Nauseas, vomits and abdominal pain had been alterations gifts in $11(12.2 \%)$ patient ones and had had to the preparation of the large bowel for the colonoscopy. No important complication was verified.

Key words: Colonoscopy, complications, sedation, accomplishment, preparation.

\section{REFERÊNCIAS}

1. Souza VCT. Colo Proctologia. $4^{a}$ edição. Ed. Medsi, 1999. p. 503-512

2. Nahas SC, Alves PA, Nabas OS, Habr-Gama A; Pinotti HW. Colonoscopia como método diagnóstico e terapêutico na doença do intestino grosso no Hospital das Clínicas da Faculdade de Medicina da Universidade de São Paulo [resumo], Rev bras Coloproct 1989; 9 (suppl. 1): 20.

3. Nahas SC, Lourenção JL, Borba M. Colonoscopia como método diagnóstico e terapêutico nas doenças do intestino grosso [resumo]. Rev bras Coloproct 1992; 12 (suppl 1): 41.

4. Nahas SC. Estudo comparativo entre os resultados obtidos com emprego do enema opaco e da colonoscopia no diagnóstico das doenças do intestino grosso [resumo]. Rev bras Coloproct 1989;9 (supl 1): 43

5. Billotta JJ, Floyd JL, Waye JD. Arterial oxygen desaturation during ambulatory colonoscopy: predictability, incidence, and clinical insignificance. Gastrointest Endosc 1990; 36 (3 Suppl): p 5-8.

6. Porro GB, Lazzaroni M. Preparation, premedication and surveillance. Endoscopy 1992; 24: p 1-8.

7. Schwesinger WH, Levine BA, Ramos R. Complications in colonoscopy. Surg Gynecol Obstet 1979; 148(2): p 270-81

8. Habr-Gama A, Rodrigues JJG, Jatobá P, Verani E, Alves PA, Goffi F, Raia A. Técnica de polipectomia transcolonoscópica. Rev Ass Méd Brasil, 1977; 23 (10): 336.
9. Waye JD. Colonoscopy: A clinical view. Mt Sinal J Med 1975; 42(1): p 1-34

10. Wolff WF, Shinya H. Polypectomy via the fiberoptic colonoscope. Removal of neoplasms beyond reach of the sigmoidoscope. N Engl J Med 1973; 288(7): p 329-32

11. Ghazi A, Grossman M. Complication of colonoscopy and polypectomy. Surg Clin North Am, 1982; 62: p 889-896.

12. Vanner SJ, MacDonald PH, Paterson WG, Prentice RS, Da Costa LR, Beck IT. A randomized prospective trial comparing oral sodium phosphate with standard polyethylene glycol-based lavage solution (golytely) in the preparation of patients for colonoscopy. Am J Gastroenterol 1991; 86 (2): p 248-9.

13. Coleman RL, Tema J. Benzodiazepínicos. Em: White PF. Tratado de anestesia venosa. Porto Alegre. Ed. ArtMed, 2001. p. 91-104

14. Bailey P, Egan T. Fentanil e congêneres. Em: White PF. Tratado de anestesia venosa. Porto Alegre. Ed. ArtMed, 2001. p. 216-248

15. V Diretrizes brasileira de Hipertensão Arterial 2006

16. Silva EJ, Câmara MAR, Gaidão E, Almeida EC. Colonoscopia: Análise crítica de sua indicação. Rev bras Coloproct 2003;23 (2): p. 77-81.

17. Habr-Gama, A, Teixeira MG, Alves PR, Ventura TC, Rodrigues, JG. Emprego de solucão de manitol a $10 \%$ no preparo do intestino grosso para colonoscopia e cirurgia. Rev. Hosp. Clin. Fac. Med. Univ. Säo Paulo 1981; 36(6): p 239-43 
18. Habr-Gama A et al. - Bowel preparation for colonoscopy: comparison of mannitol and sodium phosphate. Results of a prospective randomized study. Rev. Hosp. Clín. Fac. Med. São Paulo 1999; 54 (6): p 187-92

19. Reisine T, Pasternak G. Opioid Analgesics and Antagonists. In: Hardman JG, Limbird LE, Molinoff PB, Ruddon RW, Gilman AG, editor. Goodman and Gilman's The Pharmacological Basis of Therapeutic. $9^{\text {th }}$ ed. New York: McGraw-Hill Companies; 1996. p. 521-49

20. G \& G Rang HP, Dale MM, Ritter JM, Gardner P. Analgesic drugs. In: Livingstone C. Pharmacology. Nova York; 2003

21. Nascimento JrP, Castiglia YMM. Disritimias cardíacas per e pós-operatórias em pacientes sem doença cardiovascular. Rev bras Anestesiol 2000; 50 (5): p 350-6

22. Springman SR, Atlee JL. The etiology of intraoperative arrhythmia's. Anesthesiol Clin North Am 1989; 7: p 293-313
23. Labrunie GM, Gouveia MA. Disritmias cardíacas, cardioversão e desfibrilação. Rev bras Anestesiol 1995; 45 (1): p 47-56

24. Nahas SC, Marques CFS, Araújo SA, Aisaka AA, Nahas CSR, Pinto RA, et al. Colonoscopia como método diagnóstico e terapêutico das moléstias do intestino grosso: análise de 2.567 exames. Arq Gastroenterology 2005; 42 (2): p. 77-82

\section{Endereço para correspondência: \\ CRISTIANE JESUS SILVINO}

Alameda B, 155; Cond. Spring Village

Edf. Camélia; Apt 702

Bairro Grageru

CEP: $49.027-390$

Aracaju - SE

E-mail: crisfest@ibest.com.br 